\title{
The role of Sinonasal Surgery in Primary Ciliary Dyskinesia
}

Steven Frampton ${ }^{1}$, Woolf Walker ${ }^{1}$, Jane Lucas ${ }^{1}$, Hasnaa Ismail-Koch ${ }^{1}$, Andrea Burgess ${ }^{1}$

\section{Introduction}

Primary Ciliary Dyskinesia (PCD) describes a heterogenous group of autosomal-recessively inherited diseases which share a phenotype of abnormally functional cilia. Symptoms can vary greatly in severity depending on the particular abnormality but most cases present in childhood with respiratory disease. Some cases are associated with situs inversus. As children develop, their ENT symptoms often become more apparent, and some milder disease variants can initially present with predominantly ENT symptoms. ENT manifestations include rhinitis, rhinosinusitis, and otitis media with effusion, and their may be an increased prevalence of Obstructive Sleep Apnoea. There is increasing evidence that bacterial soiling of the lungs from the upper respiratory tract may worsen the respiratory status.

\section{Schema for Surgery}
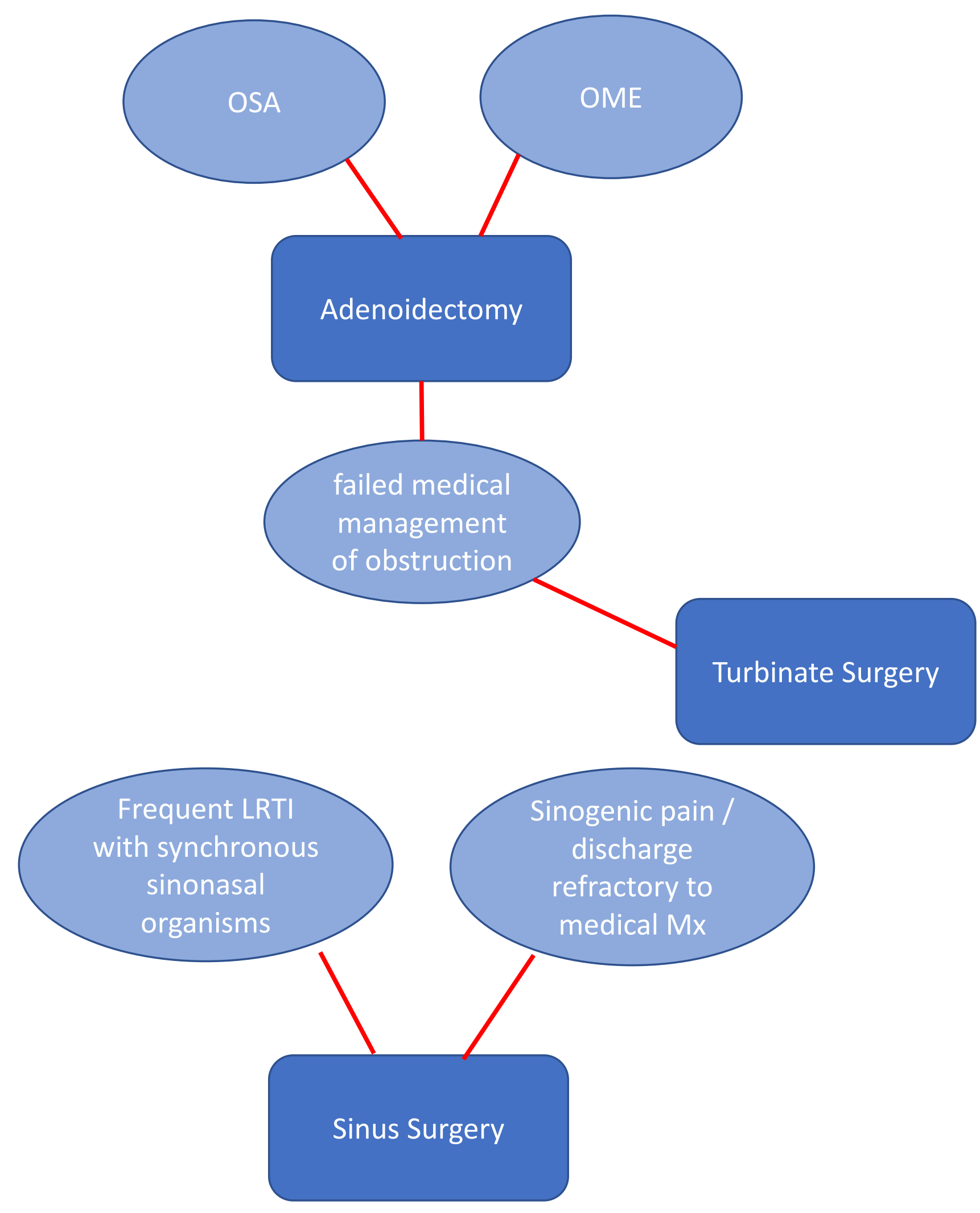

\section{Results}

From Southampton we currently provide care for 55 children with confirmed or suspected PCD. 93\% report some degree of nasal congestion and rhinorrhoea. Only $11 \%$ report headache and $9 \%$ sinus pain. None of our cohort has been identified to have sinonasal polyps.

\section{$27 \%$ of our children do not use any nasal} treatment, while $7 \%$ use behavioural techniques eg nose blowing to manage nasal secretions. $49 \%$ use saline irrigation to the nasal cavity, while an additional $11 \%$ and $6 \%$ use intranasal steroid sprays and oral antihistamine respectively.

\section{3 of our patients underwent adenoidectomy} along with tonsillectomy for OSA and 2 alongside grommets for OME (prior to PCD diagnosis) but none have required turbinate or sinus surgery in childhood. There is developing evidence that sinonasal surgical intervention may improve outcomes for the lower respiratory tract in PCD and should be considered in selected cases.

\section{Conclusion}

Based on our experience and the literature we suggest a pathway for optimising the sinonasal management of these children and minimising lower respiratory tract complications.

${ }^{1}$ Southampton Children's Hospital, University of Southampton NHS Foundation Trust

\section{Mr Steven Frampton,} Department of Otolaryngology, Tremona Road, Southampton, UK

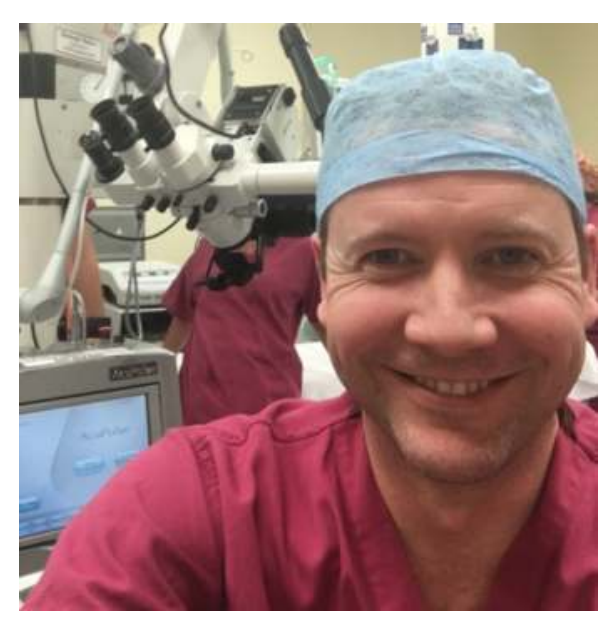

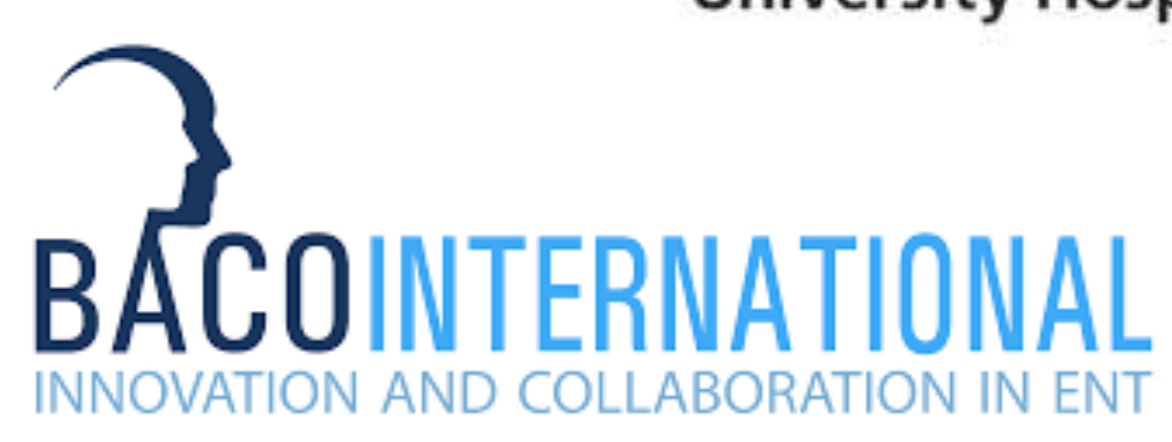

